\title{
Mobility and ESR Spectra of Free Radicals Trapped in Irradiated Urea-Poly(ethylene oxide) Complex
}

\author{
Shigetaka SHImadA, Akira FuJIWARA, and Hisatsugu KaSHIWABARA \\ Materials Research Laboratory, Nagoya Institute of Technology, \\ Showa-ku, Nagoya 466, Japan.
}

(Received January 13, 1981)

\begin{abstract}
A detailed ESR study of free radicals trapped in irradiated urea-poly(ethylene oxide) complex was carried out. On annealing at room temperature, the triplet spectrum observed immediately after $\gamma$-irradiation at $77 \mathrm{~K}$ was converted to a quartet spectrum. Using the spectrum simulation method, the quartet spectrum was found to derive from the scission free radicals, $\mathrm{H}_{\beta}$

$\mathrm{C}_{\gamma} \mathrm{H}_{3}-\mathrm{O}-\mathrm{C}_{\alpha}-\mathrm{C} \sim$, in which the methylene group took on a trans-gauche type configuration. $\mathrm{H}_{\alpha} \mathrm{H}_{\beta^{\prime}}$
\end{abstract}

Temperature dependences of hyperfine splitting widths due to $\beta$ - and $\gamma$-protons were observed and analyzed in terms of the hindered oscillation of the $\beta$-methylene group and rotation around the $\mathrm{C}_{\alpha}-\mathrm{O}$ bond axis.

KEY WORDS Poly(ethylene oxide)/Urea-PEO Complex / Free Radicals /

ESR / Mobility of Radical Site /

In previous papers, ${ }^{1,2}$ we concluded that the radical sites in urea-polyethylene complex (UPEC) were more mobile than those in polyethylene crystal by estimating the temperature dependences of ESR parameters of alkyl radicals and peroxy radicals. Our broad-line NMR study ${ }^{3}$ also clarified a much more mobile character of polyethylene molecules in the pores of urea molecules. These facts indicate that an intermolecular interaction between molecular chains is stronger in the polyethylene crystal than in UPEC due to the shorter interchain distance in the former. The mobility of the polymer chain and the intermolecular interaction are considered to influence the radiation effect in the polymer solids. Doublet and quartet ESR spectra arising from different radical species were observed in an irradiated solid of normal poly(ethylene oxide) and the urea-poly(ethylene oxide) complex (UPEOC), respectively, by Sugimoto et $a l^{4,5}$ However, the precise structure of the radical and its mobility are still opened for further study.

The aim of the present paper is to analyze the ESR spectra of free radicals produced in irradiated UPEOC and their mobility with regard to the radiation chemistry of poly(ethylene oxide) chains.

\section{EXPERIMENTAL}

Preparation of the Urea-Poly(ethylene oxide) Complex (UPEOC)

Poly(ethylene oxide) (PEO) with a weight average molecular weight of 6000 was used for this study. It is well known that the urea-poly(ethylene oxide) complex can be prepared by mixing a solution of PEO with urea. ${ }^{6,7}$ UPEOC was made in the following way. Urea $(22 \mathrm{~g})$ was added to a solution of poly (ethylene oxide) $(10 \mathrm{~g})$ in benzene $(1500 \mathrm{ml})$. This suspension was stirred in a round-bottomed flask for about one week. After filtering with suction, the powder was dried thoroughly in vacuo at $10^{-3} \mathrm{mmHg}$. UPEOC was identified by comparing its DSC curve with those of urea and poly(ethylene oxide). The melting temperature of UPEOC prepared in this study was found to be $146^{\circ} \mathrm{C}$.

\section{Irradiation Technique}

The samples were subjected to ${ }^{60} \mathrm{Co} \gamma$-irradiation at the liquid nitrogen temperature under a vacuum of $10^{-4} \mathrm{mmHg}$. The total dose was $2.8 \mathrm{Mr}$ and the dose rate $0.17 \mathrm{Mr} / \mathrm{h}^{-1}$. 


\section{ESR Measurements}

ESR spectra were recorded with a JEOL ME-2 electron spin resonance ( $\mathrm{X}$ band) spectrometer with $100 \mathrm{kHz}$ field modulation at a microwave power of $0.1 \mathrm{~mW}$. A JEOL temperature controller was used to control the temperature observation. An on line spectrum computer JEOL EC-6 was connected to the spectrometer and the data were punched out on paper tape by an A-D converter. The double integration of the first derivative curves was carried out by the electronic computer HITAC 8400 . In this way, the relative concentration of free radicals was computed.

\section{RESULTS AND DISCUSSION}

\section{Conversion of Unstable Radicals into Stable Radicals}

A triplet-like spectrum was observed at $77 \mathrm{~K}$ immediately following irradiation at the same temperature. Figure 1 shows a comparison of the spectra observed at $77 \mathrm{~K}$ before and after the heat treatment at $218 \mathrm{~K}$. It is evident that the triplet spectrum is changed into a quatet-like spectrum by warming. Figure 2 shows the variation in the relative radical concentration and the relative intensity, $I$, of the specified peak shown in Figure 1 with the temperature of the heat treatment. This specified

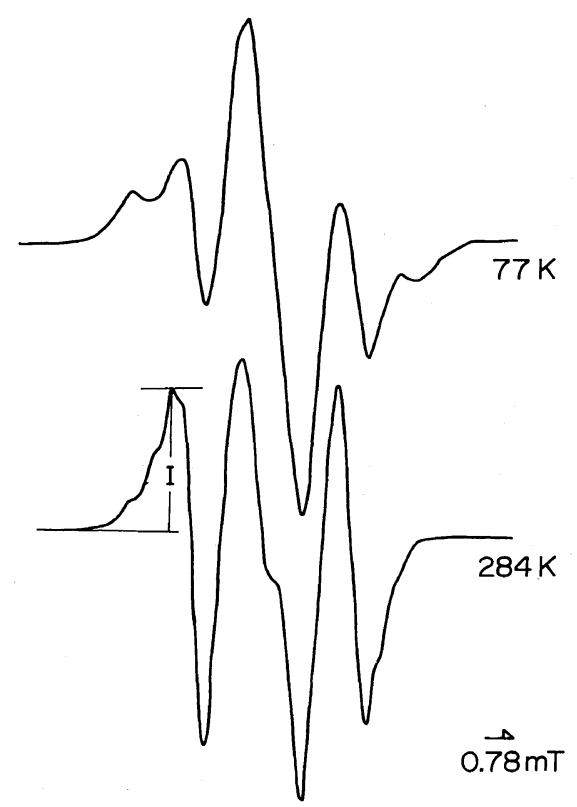

Figure 1. Change in ESR spectra at $77 \mathrm{~K}$ before and after heat treatment at $218 \mathrm{~K}$. peak is characteristic of the quartet spectrum corresponding to "stable radicals," still remaining after warming at $320 \mathrm{~K}$. The spectra, which were the basic data for obtaining Figure 2, were observed at $77 \mathrm{~K}$ after heat treatment at respective temperatures. In spite of the increase in the specified peak intensity with temperature, the total concentration of free radicals shows no remarkable decrease with temperature. This fact indicates that the major parts of the free radical corresponding to triplet spectrum, which we call "unstable radicals," are converted into more stable radicals by warming. In other words, it may be concluded that the unstable radicals are the precursor of the stable radicals.

\section{Assignment of the Stable Free Radicals}

Figure 3 shows the ESR spectra of stable free

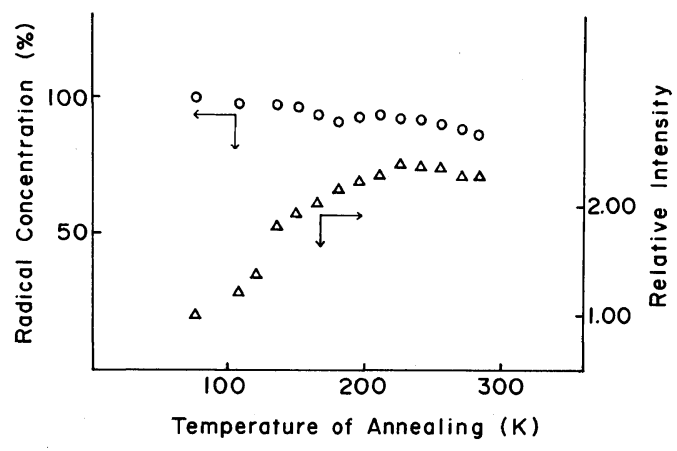

Figure 2. Change in the relative radical concentration $(O)$ and the relative intensity $(I)$ of the specified peak indicated by arrow in Fig. $1(\triangle)$.

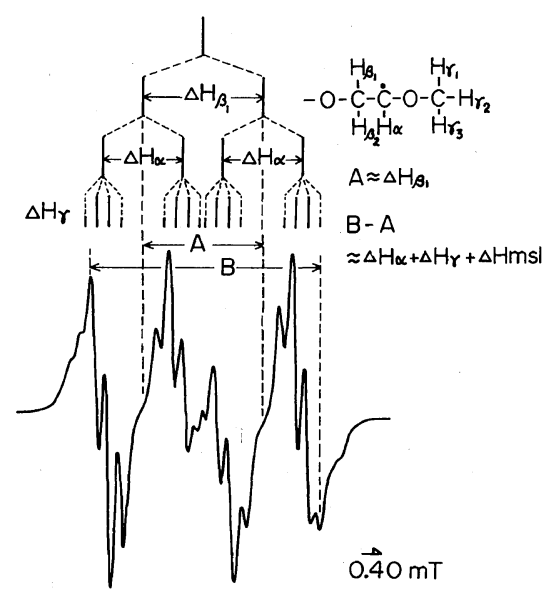

Figure 3. ESR spectra of stable radicals trapped in UPEOC observed at $293 \mathrm{~K}$. 
radicals observed at room temperature. This seems to be a quartet spectrum with the approximately equal intensities, each component of which is composed of quartet subsplitting with a binominal intensity. The main quartet is considered to be caused by hyperfine splittings due to two protons,

$$
\begin{aligned}
& \mathrm{H}_{\beta_{1}} \text {. } \\
& \mathrm{H}_{\alpha} \text { and } \mathrm{H}_{\beta_{1}} \text { in ether type free radicals, } \sim \mathrm{C}-\mathrm{C}-\mathrm{O} \sim \text {, } \\
& \mathrm{H}_{\beta_{2}} \mathrm{H}_{\alpha}
\end{aligned}
$$

because the orders of magnitude of the values, 1.8 and $2.5 \mathrm{mT}$ are similar to the values of splittings due to $\alpha$ and $\beta$ protons of the similar radical reported in the other papers. ${ }^{8,9}$ As discussed in the next section, hyperfine splitting due to $\mathrm{H}_{\beta_{2}}$ proton should be canceled out. On the other hand, the value of quartet subsplitting is $c a .0 .25 \mathrm{mT}$ which is also in good agreement with the value of hyperfine splitting due to $\gamma$-proton of the ether type free radical, $\mathrm{H}_{\gamma}$

$\sim \dot{\mathrm{C}}-\mathrm{O}-\mathrm{CH}_{\gamma}{ }^{9} \quad$ Consequently, the quartet-

$$
\mathrm{H}_{\alpha} \quad \mathrm{H}_{\gamma}
$$

quartet spectrum is considered to be that of the scis-

$$
\mathrm{H}
$$

sion type radical, $\sim \mathrm{C}-\mathrm{C}-\mathrm{O}-\mathrm{CH}_{3}$. The stick

$$
\mathrm{H} \quad \mathrm{H}
$$

diagram in Figure 3 is a good representation of the assignment. The extralines observed outside the quartet-quartet spectrum is caused by an anisotropic hyperfine splitting due to $\alpha$-proton;

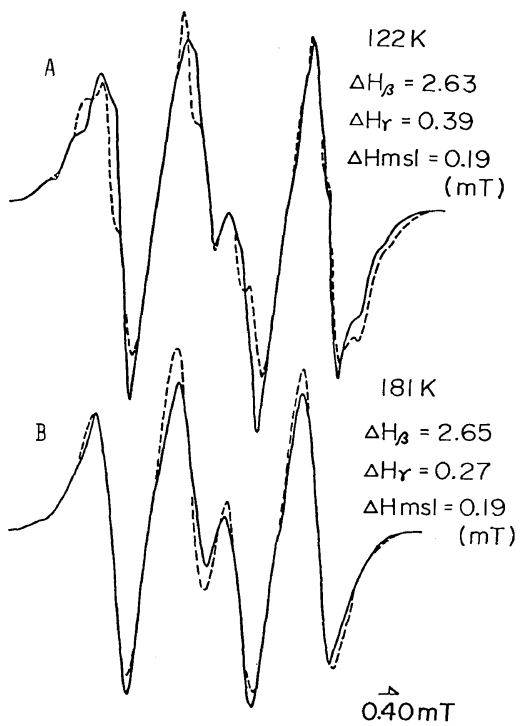

it shows a so called amorphous pattern.

In order to certify the above assignment, the computer simulation was carried out under following conditions: (a) We assumed that the anisotropy of the coupling constant due to $\alpha$-proton is axially symmetric and that of coupling constant due to $\beta$ proton is negligible. (b) The rough values of coupling constant due to $\alpha, \beta$ and $\gamma$-protons, $\Delta H_{\alpha}, \Delta H_{\beta}$, and $\Delta H_{\gamma}$ and line width, $\Delta H m s l$, are estimated from the values of $A$ and $B$ represented in Figure 3. The anisotropic hyperfine splitting due to $\alpha$-proton, $A_{\|}$ and $A_{\perp}$ were also estimated to be 2.5 and $1.5 \mathrm{mT}$, respectively. The orders of magnitude of these values are similar to those obtained by another workers. ${ }^{9}$ By using the values of spectral parameters described previously, we could obtain theoretical spectra assuming that each of the components is gaussian line in shape. Several theoretical spectra were calculated by gradually changing the values of spectral parameters. These spectra were recorded on an $\mathrm{X}-\mathrm{Y}$ plotter and compared with the observed spectra in order to get the best fit.

The experimental spectrum observed at $293 \mathrm{~K}$ and the calculated spectrum shown in Figure 4(C) seems the best fit with respect to the whole line shape and peak positions. Consequently, it can be concluded that stable radicals is the scission type radical, $\mathrm{H}_{\beta} . \quad \mathrm{H}_{\gamma}$ $-\mathrm{C}-\dot{\mathrm{C}}-\mathrm{O}-\mathrm{CH}_{\gamma}$. It can also be assumed that $\mathrm{H}_{\beta^{\prime}} \mathrm{H}_{\alpha} \quad \mathrm{H}_{\gamma}$
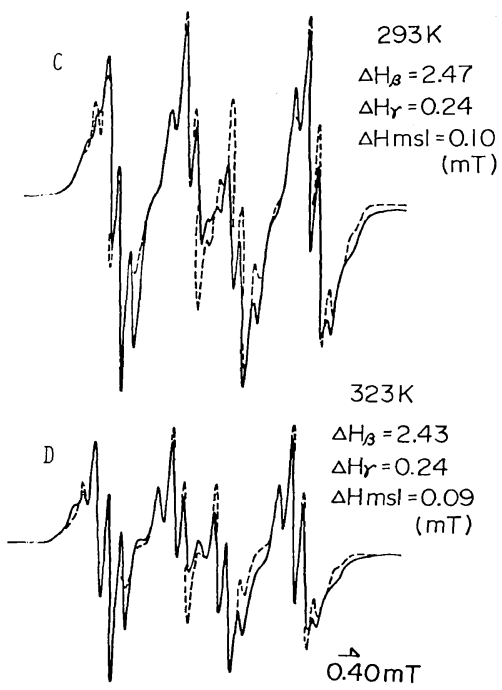

Figure 4. Change in ESR spectra of stable radicals trapped in UPEOC with observation temperature: - 
Table I. Possibile free radicals produced in irradiated poly(ethylene oxide),

$\begin{array}{lll}\mathrm{H} \mathrm{H}_{1} & \mathrm{H} & \mathrm{H}\end{array}$

$\sim \mathrm{C}-\mathrm{C}_{1}-\mathrm{O}_{2}-\mathrm{C}_{3}-\mathrm{C}_{4}-\mathrm{O} \sim$

$\begin{array}{lll}\mathrm{H} & \mathrm{H} & \mathrm{H}\end{array}$

\begin{tabular}{|c|c|c|c|c|}
\hline $\begin{array}{c}\text { Destructed } \\
\text { chemical } \\
\text { bond }\end{array}$ & \multicolumn{2}{|c|}{$\begin{array}{l}\text { Primarily produced } \\
\text { free radicals }\end{array}$} & \multicolumn{2}{|l|}{$\begin{array}{c}\text { Secondarily } \\
\text { produced } \\
\text { radicals }\end{array}$} \\
\hline $\mathrm{C}_{1}-\mathrm{H}_{1}$ & $\begin{array}{c}\mathrm{H} \quad \mathrm{H} \\
\sim \mathrm{C}-\mathrm{C}-\mathrm{C}-\mathrm{C} \sim \\
\mathrm{H} \mathrm{H} \quad \mathrm{H}\end{array}$ & (a) & & \\
\hline $\mathrm{C}_{1}-\mathrm{O}_{2}$ & $\begin{aligned} & \mathrm{H} \mathrm{H} \\
\sim & \mathrm{C}-\mathrm{C}-\mathrm{O} . \\
& \mathrm{H} \mathrm{H}\end{aligned}$ & (b) $\rightarrow$ & $\begin{aligned} & \mathrm{H} \\
\sim & \mathrm{C}-\dot{\mathrm{C}}-\mathrm{OH} \\
& \mathrm{H} \mathrm{H}\end{aligned}$ & (d) \\
\hline & $\begin{array}{r}\mathrm{H} \mathrm{H} \\
\sim \mathrm{O}-\mathrm{C}-\mathrm{C} \cdot \\
\mathrm{H} \mathrm{H}\end{array}$ & $(\mathrm{c}) \rightarrow$ & $\begin{array}{c}\mathrm{H} \\
\sim \mathrm{O}-\dot{\mathrm{C}}-\mathrm{CH} \\
\mathrm{H} \mathrm{H}\end{array}$ & (e) \\
\hline $\mathrm{C}_{3}-\mathrm{C}_{4}$ & $\begin{array}{cc}\mathrm{H} \mathrm{H} & \mathrm{H} \\
-\mathrm{C}-\mathrm{C}-\mathrm{O}-\mathrm{C} \\
\mathrm{H} \mathrm{H}\end{array}$ & $(f) \rightarrow$ & $\begin{array}{cc}\mathrm{H} & \mathrm{H} \\
-\mathrm{C}-\dot{\mathrm{C}}-\mathrm{O}-\mathrm{CH} \\
\mathrm{H} \mathrm{H} & \mathrm{H}\end{array}$ & (g) \\
\hline
\end{tabular}

during $\gamma$-irradiation three types of chemical degradations of poly(ethylene oxide) chain,

$$
\begin{array}{llll}
\mathrm{H} & \mathrm{H} & \mathrm{H} & \mathrm{H}
\end{array}
$$

$-\mathrm{C}-\mathrm{C}_{1}-\mathrm{O}_{2}-\mathrm{C}_{3}-\mathrm{C}_{4}-$, occur and then var-

$\begin{array}{llll}\mathrm{H} & \mathrm{H}_{1} & \mathrm{H} & \mathrm{H}\end{array}$

ious free radicals are produced. We can also assume that less stable radicals are converted into more stable radicals as shown in Table $\mathbf{I}$. Since the stable radicals observed in the present study are attributed to the scission type radical, (g), destruction of the chemical bond $\mathrm{C}_{3}-\mathrm{C}_{4}$ during $\gamma$-irradiation is very likely. Unstable radical species which convert to the stable radicals, $(\mathrm{g})$, is not identified completely. However, the unstable radical species possibly is scission type radical, (f), because of the triplet spectrum. Consequently, it is reasonable to consider that the radical conversion mentioned in the previous section is brought about by the migration of the proton,<smiles>COCCOC</smiles>

Structure of Scission Type Free Radicals in UPEOC

It is well known that the configuration of a free radical site can be clarified by estimating the values of hyperfine splitting due to $\beta$-protons. In the present section, we discuss again the assignment of the stable radical in view of the values of hyperfine splitting due to $\beta$-protons of free radicals trapped in UPEOC and demonstrate its structure with relation to the conformation of poly(ethylene oxide) chains.

The hyperfine splitting due to $\beta$-protons of the neighbouring methylene group in the radical, $\mathrm{H}_{\beta_{1}}$.

$-\mathrm{C}-\dot{\mathrm{C}}-\Delta H_{\beta_{i}}$, can be considered to arise in two $\mathrm{H}_{\beta_{2}} \mathrm{H}_{\alpha}$

ways; i) by $\pi-\delta$ polarization involving the spin polarization of $s p^{2}$-orbital on the $\beta$-carbon and ii) by hyperconjugative interaction with the $p$ orbital on the $\alpha$-carbon. These mechanism are represented as the first and second terms, respectively, in the following equation by Heller and McConnell, ${ }^{10}$

$$
\begin{aligned}
\Delta H_{\beta_{i}} & =A+K \rho \cos ^{2} \theta_{i} \\
& =A+B \cos ^{2} \theta_{i}
\end{aligned}
$$

where $K$ is a constant, $\rho$ is the spin density, and $\theta_{i}$ is the projected angle between the $\mathrm{C}_{\beta i}-\mathrm{H}_{\beta i}$ bond and the $\pi$-electron axis on the plane perpendicular to the direction of the $\mathrm{C}_{\alpha}-\mathrm{C}_{\beta}$ bond.

Buley et al. ${ }^{11}$ found the small splitting $(0.14 \mathrm{mT})$ to be due to the aldehydic proton, $\mathrm{H}_{\beta_{1}}$, in the radical, $\mathrm{CH}_{2} \cdot(\mathrm{OH}) \cdot \dot{\mathrm{C}}_{\alpha} \mathrm{H}_{\alpha}-\mathrm{C}_{\beta_{1}} \mathrm{H}_{\beta_{1}} \mathrm{O}$, in which the aldehydic group takes a plane perpendicular to the $p$-orbitals; i.e., $\theta_{\beta_{1}}=90^{\circ}$. Hence, these authors concluded that the spin polarization mechanism should lead to a splitting only of the order of $0.1 \mathrm{mT}$ with consideration of the splitting due to methyl protons in the acetonyl radical, $\mathrm{CH}_{2}-\mathrm{C}-\mathrm{CH}_{3}$, and thus the $\mathrm{O}$

hyperconjugation interaction was neglected. The spin density at $\beta$-carbon of the ether type radical,

$$
\underset{\sim}{\sim} \underset{\mathrm{H}_{\beta_{2}} \mathrm{H}_{\alpha}}{\stackrel{\mathrm{C}}{\beta_{\beta_{1}}}} \dot{\mathrm{C}}_{\alpha}-\mathrm{O} \sim \text {, in which the methylene group takes }
$$

on a tetrahedral conformation, is less than that in the case of the radical, $\mathrm{CH}_{2}(\mathrm{OH}) \dot{\mathrm{C}}_{\alpha} \mathrm{H}_{\alpha}-\mathrm{C}_{\beta_{1}} \mathrm{H}_{\beta_{1}} \mathrm{O}$. Consequently, the spin polarization mechanism in the hyperfine splitting due to the $\beta$-proton of the ether type radical can not be neglected completely. Thus eq 1 may, be simplified as follows:

$$
\Delta H_{\beta i}=K \rho \cos ^{2} \theta_{i}=B \cos ^{2} \theta_{i}
$$

The hyperfine splitting widths due to the two $\beta$ - 


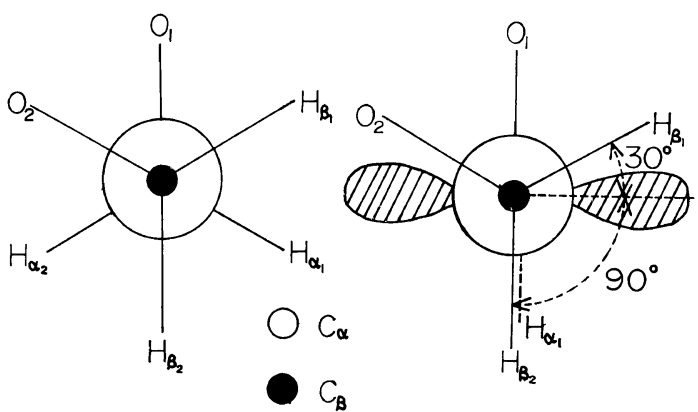

$\Delta H_{\beta}=B_{\rho} \cos ^{2} \theta$

Figure 5. Schematic illustration of steric configuration of the atoms at the site of the stable radical.

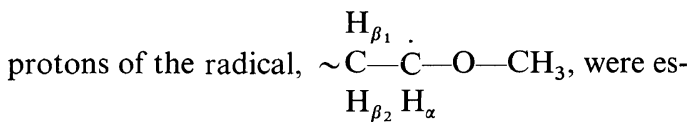
timated to be 2.62 and $0.0 \mathrm{mT}$ in the previous section. However, if the stable free radicals are assum-

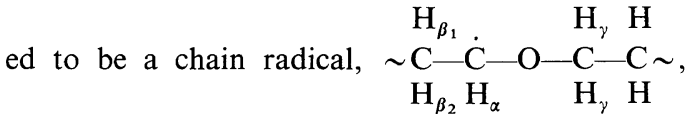
the value of one of the hyperfine splittings due to $\beta$-protons should be of the order of magnitude of the coupling constant due to the $\gamma$-proton, $0.24 \mathrm{mT}$. That is, subsplitting quartet of experimental spectra can be considered as arising from splittings due to two $\gamma$-protons and one $\beta$-proton is the chain radical. These assumed values of $\Delta H_{\beta_{1}}$ and $\Delta H_{\beta_{2}}$, when substituted into eq 2 , give the following relations,

$$
\begin{aligned}
\Delta H_{\beta_{1}} & =2.62=B \cos ^{2} \theta_{1} \\
\Delta H_{\beta_{2}} & =0.24=B \cos ^{2} \theta_{2}
\end{aligned}
$$

Since the $\beta$-methylene group is in the regular tetrahedral arrangement, eq 5 is satisfied as shown in Figure 5,

$$
\theta_{2}-\theta_{1}=60^{\circ}
$$

Equations 3, 4 and 5 lead to eq 6,

$$
\frac{\cos ^{2} \theta_{1}}{\cos ^{2}\left(60^{\circ}+\theta_{1}\right)}=10.9
$$

and the following values were also obtained,

$$
\begin{aligned}
& \theta_{1}=13.0^{\circ} \\
& \theta_{2}=73.0^{\circ} \\
& B=2.76 \mathrm{mT}
\end{aligned}
$$

The value of $B$ obtained in eq 9 should be less probable, being much smaller than $3.92-5.0 \mathrm{mT}$ obtained in the studies of the aliphatic radicals carried out so far. ${ }^{12-16}$ Griffith $^{9}$ also estimated the value of $B$ to be $4.17 \mathrm{mT}$ in the case of the ether type radical, $\sim \mathrm{CH}_{2}-\mathrm{O}-\dot{\mathrm{C}}_{\alpha} \mathrm{H}_{\alpha}-\mathrm{CH}_{3}$, by analysing the hyperfine splitting width of $\beta$-methylene protons. Hence, it is reasonable that the stable radical should not be assigned to be the chain type radical, $\mathrm{H}_{\beta_{1}}$. $\quad \mathrm{H}_{\gamma} \mathrm{H}$

$\sim \mathrm{C}-\mathrm{C}-\mathrm{O}-\mathrm{C}-\mathrm{C}-$, but to be the scission type $\mathrm{H}_{\beta_{2}} \mathrm{H}_{\alpha} \quad \mathrm{H}_{\gamma} \mathrm{H}$

$\mathrm{H}_{\beta_{1}} . \quad \mathrm{H}_{\gamma}$

radical, $-\mathrm{C}-\mathrm{C}-\mathrm{O}-\mathrm{CH}_{\gamma}$, as concluded in the $\mathrm{H}_{\beta_{2}} \mathrm{H}_{\alpha} \quad \mathrm{H}_{\gamma}$

previous section. In addition, it was concluded that the hyperfine splitting widths of the radical, $\mathrm{H}_{\beta_{1}}$

$\sim \mathrm{C}-\dot{\mathrm{C}}-\mathrm{O}-\mathrm{CH}_{3}$, have ca. 2.62 and $0.0 \mathrm{mT}$. $\mathrm{H}_{\beta_{2}} \mathrm{H}_{\alpha}$

These values were substituted into eq 2 and the angles, $\theta_{1}$ and $\theta_{2}$, were found to be $30^{\circ}$ and $90^{\circ}$, respectively. As a result, the structure of the stable radical $(\mathrm{g})$ can be picturized as shown in Figure 5. It is well known that the conformation of polyethyleneoxide chains in normal crystalline phase are of trans-trans-gauche type ${ }^{17}$; i.e., a 72 -helical structure as represented in Figure 5. Since this conformation is in good agreement with that of the free radical $(\mathrm{g})$, it can be said that the $7_{2}$-helical structure of poly(ethylene oxide) chain still exists in irradiated UPEOC.

\section{Variation of ESR Spectra of Scission Type Radicals Trapped in UPEOC}

Figure 4 shows the change of ESR spectra of stable radicals produced in irradiated UPEOC with the temperature of observation. The ESR spectra of stable radicals changed remarkably and sharper spectra were observed when the observation temperature was about $200 \mathrm{~K}$. Because of this motional narrowing of line widths, subsplittings due to $\gamma$ proton were well resolved at temperatures higher than $200 \mathrm{~K}$.

We can see that subsplittings are also observed at lower temperature, $122 \mathrm{~K}$, as shown in Figure 4(A). However, at an intermediate temperature, $181 \mathrm{~K}$, the subsplitting disappears as shown in Figure 4(B). This seems to result from some very complicated temperature dependences of the ESR parameters, $\Delta H_{\alpha}, \Delta H_{\beta}, \Delta H_{y}$ and $\Delta H m s l$. For example, it may be 


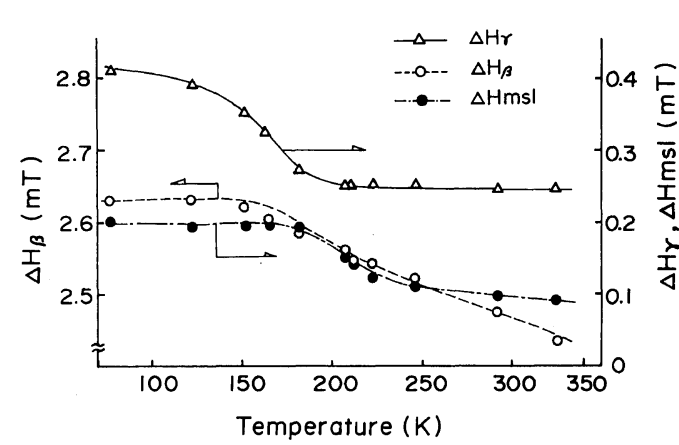

Figure 6. Hyperfine splitting widths due to $\beta$ - and $\gamma$ protons and line width of the stable radicals $v s$. temperature of observation: $\triangle(--), \Delta H_{\gamma} ; \bigcirc(--), \Delta H_{\beta} ; \mathbf{O}^{(--)}$, $\Delta H m s l$.

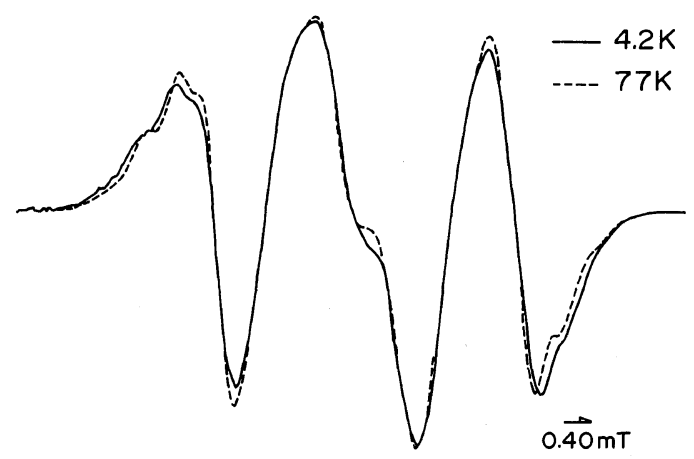

Figure 7. Comparison of the ESR spectra of the stable radical observed at $77 \mathrm{~K}(--)$ and at $4.2 \mathrm{~K}(-)$.

considered that the ratio of $\Delta H_{\gamma}$ to $\Delta H m s l$ determines the resolution of subsplitting due to $\gamma$ protons. By the computer simulation method described in the previous section, the ESR parameters at various temperatures were determined.

$\Delta H_{\alpha}$ does not show any detectable decrease throughout the temperature range studied. Figure 6 shows the variation in the values of $\Delta H_{\dot{\beta}}, \Delta H_{\gamma}$ and $\Delta H m s l . \Delta H_{\beta}$ does not change until $150 \mathrm{~K}$ and then it abruptly decreases above this temperature. On the other hand, $\Delta H_{\gamma}$ begins to decrease at a very low temperature compared with the curve for $\Delta H_{\beta}$, indicating that $\gamma$-protons are quite mobile even at the temperature of liquid nitrogen.

As will be discussed in detail in the next subsection, hyperfine splitting decreases with rising temperature and $\Delta H_{\gamma}$ seems to increase at a temperature lower than $77 \mathrm{~K}$. In connection with this matter, the spectrum of a stable redical was ob- served also at liquid helium temperature. A comparison of the spectra observed at $4 \mathrm{~K}$ and $77 \mathrm{~K}$ is shown in Figure 7. According to the discussion on the stick diagram of the spectrum (Figure 3), the outer shoulders of the main quartet correspond to the values of $\Delta H_{\gamma}$. The spectrum observed at $4 \mathrm{~K}$ appears to have a slightly broader $\Delta H_{\gamma}$. Thus, it may be said that the difference in the spectra observed at $4 \mathrm{~K}$ and $77 \mathrm{~K}$ reflects the difference in the mobility of the radical sites at these temperatures. A detailed discussion on the spectral change at the temperatures below $77 \mathrm{~K}$ will be presented in a future study.

\section{Molecular Motion of the Scission Type Radical in UPEOC}

Recently, the effect of hindered rotation of the molecular site on the localization of the unpaired electron was discussed by Bullock and Howard ${ }^{18,19}$ in the case of the cation of 1,2,4,5-tetrahydroxybenzene and other related compounds. These authors discussed the temperature dependence of hyperfine splitting and suggested that the value of the hyperfine coupling constant due to the proton of hydroxyl group depends on the spin density of the carbon atom bonded to the oxygen and the spin polarization. The latter is related either to the twisted angle of the plane of the benzene ring or the value of the overlap integral of $p$-orbitals on the oxygen and carbon atoms. We also made similar considerations in this regards.

Rotation about the axis of the $\mathrm{C}_{\alpha}-\mathrm{O}$ bond in the $\mathrm{H}_{\gamma} \quad . \quad \mathrm{H}_{\beta}$

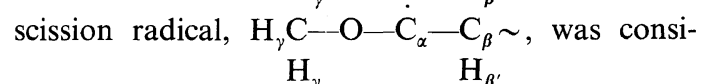
dered. This rotation results in the delocalization of the unpaired electron at the oxygen atom, and leads to smaller hyperfine splitting due to $\gamma$-protons. These interpretations fit well with experimental results on the temperature dependence of the hyperfine splittings due to $\gamma$-protons. The temperature dependence of the coupling constant due to $\beta$ proton shown in Figure 6 is interpreted in terms of the exchange of two $\beta$-protons due to the hindered oscillation about the $\mathrm{C}_{\alpha}-\mathrm{C}_{\beta}$ bond.

When $\theta_{0}$ and $\Delta \theta$ are defined as the equilibrium angle and the displacement due to torsion, the average value of the coupling constant, $\left\langle\Delta H_{\beta}\right\rangle$ due to $\beta$-proton is expressed by the following equation. ${ }^{1}$ 


$$
\begin{aligned}
\left\langle\Delta H_{\beta}\right\rangle= & B \rho\left\langle\cos ^{2} \theta\right\rangle=B \rho\left\langle\cos ^{2}\left(\theta_{0}+\Delta \theta\right)\right\rangle \\
= & B \rho\left[\cos ^{2} \theta_{0}\left\langle\cos ^{2} \Delta \theta\right\rangle\right. \\
& \left.+\sin ^{2} \theta_{0}\left(1-\left\langle\cos ^{2} \Delta \theta\right\rangle\right)\right]
\end{aligned}
$$

As stated in the previous section, $\theta_{0}$ was found to be $30^{\circ}$. Substituting this value, $\theta_{0}=30^{\circ}$, into eq 10 , we obtained,

$$
\left\langle\Delta H_{\beta}\right\rangle=B \rho\left(1 / 4+(1 / 2) \cos ^{2} \Delta \theta\right)
$$

$\left\langle\Delta H_{\beta}\right\rangle$ in eq 10 or eq 11 decreases with increasing temperature since the amplitude of torsional oscillation, $\Delta \theta$, increases with increasing temperature. This character is well shown by experimental facts. Based on this discussion with regard to the motions of $\beta$ - and $\gamma$-protons, it may be concluded that $\gamma$ protons are more mobile than $\beta$-protons in the $\mathrm{H} \mathrm{H}$

scission type radical, $\mathrm{CH}_{3}-\mathrm{O}-\mathrm{C}-\mathrm{C} \sim$. It should $\mathrm{H}$

be said that the temperature dependence of $\Delta H_{\beta}$ and $\Delta H_{\gamma}$ was observed separately in our study, meaning that, in this paper, it has been possible to make a separate discussion of the local mobilities of methylene and methyl groups.

Acknowledgement. The partial support by a Grant-in-Aid for Scientific Research from the Ministry of Education, Science and Culture of Japan is greatly acknowledged.

\section{REFERENCES}

1. S. Shimada, Y. Hori, and H. Kashiwabara, Polymer, 19, 763 (1978).

2. Y. Hori, S, Shimada, and H. Kashiwabara, Polymer, 18, 1143 (1977).

3. Y. Hori, T. Tanigawa, S. Shimada, and $\mathrm{H}$. Kashiwabara, Polym. J., 13, in press (1981).

4. I. Nitta, S. Ohnishi, and Y. Nakajima, Annu. Rep. JARRP, 3, 297 (1961).

5. S. Sugimoto, S. Ohnishi, and I. Nitta, Annu. Rep. JARRP, 8, 177 (1966).

6. F. E. Bailey and H. G. France, J. Polym. Sci., 49, 397 (1961).

7. H. Tadokoro, T. Yoshihara, Y. Chatani, and S. Murahashi, J. Polym. Sci., B, 2, 363 (1964).

8. C. Corvaja, M. Brustolon, and G. Giacometti, Z. Phis. Chem., 66, 279 (1969).

9. O. H. Griffith, J. Chem. Phys., 42, 2651 (1965).

10. C. Heller and H. M. McConnell, J. Chem. Phys., 32, 1535 (1960).

11. A. L. Buley, R. O. C. Norman, and R. J. Pritehett, J. Chem. Soc., Sect B, 849 (1966).

12. C. Corvaja, J. Chem. Phys., 44, 1958 (1966).

13. O. H. Griffith, J. Chem. Phys., 42, 2651 (1965).

14. O. H. Griffith, J. Chem. Phys., 41, 1093 (1964).

15. R. W. Fessenden and P. H. Schuler, J. Chem. Phys., 39, 2147 (1963).

16. I. Miyagawa and K. Itoh, J. Chem. Phys., 36, 2957 (1962).

17. Y. Wada "Physical Properties of High Polymers," Baifukan Book Co. Ltd., Tokyo, 1971.

18. A. T. Bullock and C. H. Howard, Mol. Phys., 27, 949 (1974).

19. A. T. Bullock and C. H. Howard, J. Magn. Resonance, 25, 47 (1977). 